\title{
DISEÑO DE UN CEPILLO DENTAL DESECHABLE Y BIODEGRADABLE
}

${ }^{1}$ Nelly Mercedes Rivas Gómez, ${ }^{2}$ Ciro Alfonso Reyes Álvarez, ${ }^{3}$ Diana $M$. Camargo L.

${ }^{1}$ Odontóloga, U. Santo Tomás. 2Diseñador Industrial, U.I.S.

${ }^{3}$ M.SC. Epidemiología, Docente, U. Santo Tomás.

Autor responsable de correspondencia: Dra. Diana M. Camargo L.

e-mail: diana_nico@ustabuca.edu.co

RESUMEN

Objetivo: Diseñar un cepillo dental desechable y biodegradable que, además de proporcionar un mecanismo de higiene oral eficiente fuera del hogar, contribuyera a preservar el medio ambiente.

Materiales y Métodos: Se diseñaron y elaboraron 30 cepillos dentales con mango de madera y cerdas de fibras naturales, de fique, impregnadas con crema dental. El mango lleva en su punta un receptáculo, que contiene, en su interior, unas semillas de árbol maderable, para preservar el material con que está fabricado. Para efectuar las pruebas se escogieron 10 estudiantes de último semestre de odontología y 20 personas de diferentes ocupaciones; se probaron los cepillos y se efectuaron las encuestas que evaluaron la aceptación general, presentación y utilización.

Resultados: Se obtuvo un grado de aceptación general superior al 90\%, en cuanto a presentación. Como producto ecológico su aceptación fue del 100\%. La totalidad de la población lo compraría y con algunos ajustes en cuanto a diseño del mango y las cerdas, se convertiría en una alternativa para promover la salud oral y la preservación del ciclo biológico del ecosistema, en general.

Conclusión: El producto cumplió con las expectativas de higiene oral y preservación del medio ambiente. [Rivas NM, Reyes CA, Camargo DM. Diseño de un cepillo dental desechable y biodegradable. Ustasalud 2002;1:19-25]

PAI.ABRAS CLAVES: Cepillo dental, Material biodegradable, Higiene oral.

\section{A Biodegradable Toothbrush Design}

\begin{abstract}
Purpose: The purpose of this investigation was to develop a disposable toothbrush. I

Material and Methods: Thirty toothbrushes were designed and manufactured with a wooden handle and natural sisal bristles soaked in toothpaste. The handle had a receptacle on the end containing seeds for timber-yielding trees to preserve the material it was manufactured in. In order to carry out the tests, 10 students in the final semester of dentistry and 20 people with different occupations were chosen. The toothbrushes were tested and the corresponding surveys were applied with a general degree of acceptance of over $90 \%$ in regard to the toothbrush's presentation.

Results: As an ecological product, it was $100 \%$ accepted. The whole population surveyed would buy it and with a few adjustments as far as the design of the handle and bristles, they would consider it an alternative to promote oral health and the preservation of the biological cycle of the ecosystem in general.
\end{abstract}

Conclusion. The product met the expectations for oral hygiene and environmental conservation.

KEY WORDS: Toothbrush, Biodegradable, Oral hygiene 


\section{INTRODUCCIÓN}

Las diferentes culturas antiguas, como la de los Mayas, Fenicios, Etruscos y Egipcios, utilizaron para su higiene oral, plantas y esencias medicinales. A pesar de que no era el procedimiento más adecuado, por la falta de instrumentos, este indicio se marcó como el principio de la higiene oral. ${ }^{(1)}$

El rostro es una parte importante de nuestro cuerpo, ya que por él nos comunicamos con los demás. En él, la boca es una parte primordial para dar una buena impresión, ya que una boca limpia es una boca sana. ${ }^{(2)}$

Uno de los principales y más comunes problemas en la cavidad oral es la placa dentobacteriana, capa delgada, incolora y pegajosa que se forma en todo momento del día; es abundante en bacterias, que producen ácidos y otras sustancias agresivas para la boca, debilitando el esmalte y contribuyendo a la producción de caries y enfermedad periodontal. (2)

La remoción de la placa regular y completa es un prerrequisito para una buena salud oral. Por lo cual el cepillado dental, después de cada comida, es fundamental para eliminar los residuos alimenticios y la placa dentobacteriana. En este aspecto, el cepillo dental cumple dos propósitos: Remover la placa y ser un vehículo para la crema dental, elemento que aumenta la remoción extrínseca de la placa dental. ${ }^{(3-5)}$

\section{Cepillos de dientes}

Existe una gran diversidad de cepillos de dientes en el mercado; quizás la mayor diferencia actual está marcada por los cepillos manuales y eléctricos. Una revisión reciente, evalúa los efectos y utilidad de estos últimos comparándolos con los cepillos manuales. Aparte de las diferencias en cuanto a diseño experimental e indicadores de evaluación para la remoción de placa, parece confirmarse su mayor efectividad. ${ }^{(6)}$

A pesar de esto, otros estudios han concluido que no son superiores y que el factor dominante para la remoción de placa es la destreza del usuario. (7)
Los cepillos de dientes manuales siguen utilizándose ampliamente. Son una ayuda efectiva para limpiar las superficies lisas y oclusales, con excepción del sistema de fisuras, para el cual deberán utilizarse otros métodos de limpieza. ${ }^{(3)}$

La efectividad del cepillo de dientes manual depende de que el usuario guíe la cabeza hacia todas las superficies del diente y provea una apropiada acción mecánica para que los filamentos remuevan los depósitos de la superficie. $^{(8)}$

La elección del material de las cerdas no tiene mucha importancia respecto de la eficiencia de la limpieza. No obstante, son preferibles los filamentos sintéticos, por tener mejores propiedades físicas e higiénicas, fortaleciendo de esa manera la calidad y duración del cepillo. En la actualidad hay escasez de cerda natural, la calidad es muy inconsistente y su comportamiento cambia rápidamente de condiciones secas a mojadas y a temperaturas variables. $^{(3)}$

La posición y densidad de los filamentos (derechos, multipenachos, en forma de V, dentados u otros), no tiene influencia importante en la eficiencia de la limpieza. Se ha comprobado que ninguna de las formas existentes puede remover con efectividad la placa interproximal y tampoco parece existir una correlación entre la dureza de los penachos y la eficiencia del cepillo para limpiar las superficies anchas de los dientes. (3) Dyer y col., demostraron que la abrasión sobre la dentina no depende de la dureza de los filamentos sino de la cantidad de crema dental atrapada en los diferentes tipos de filamentos, la que ocasiona la lesión dentinal. ${ }^{(9)}$

Adicionalmente, las puntas redondeadas sumadas a una buena técnica de cepillado también parecen disminuir la abrasión dentinal y el daño gingival. ${ }^{(10)}$ Sin embargo, según el estudio realizado por Checchi y col., son pocos los cepillos disponibles en el mercado, y en bajo porcentaje, los que cumplen este criterio de calidad aceptable. (11)

Entre las variaciones normales, el largo y ancho del cabezal de los cepillos de dientes convencionales no ejercen influencia alguna sobre la superficie de la limpieza o el acceso a cualquier superficie dentaria. ${ }^{(3,7)}$ 
La forma del mango no tiene en sí ninguna influencia sobre la superficie de limpieza del cepillo de dientes; no obstante, su forma puede ser importante para que el cepillado sea más cómodo. Cabe resaltar que hay muy poca investigación en este campo. ${ }^{(3)}$

Los cepillos de dientes desgastados no pueden efectuar una buena limpieza de dientes y pueden lastimar la encía. Por lo general, los cepillos de dientes deben cambiarse cada 3 ó 4 meses. El cepillo de dientes puede limpiar sólo 102 dientes por vez. ${ }^{(3)}$

\section{Técnicas de cepillado}

Para una buena higiene oral se debe hacer un cepillado mínimo tres veces al día, una vez después de cada comida usando una buena técnica de cepillado y un buen cepillo con cerdas suaves, recordando cepillar las superficies externas, internas, masticatorias y la lengua. ${ }^{(2)}$

Idealmente, las pruebas de seguridad y eficacia con cepillos dentales deben realizarse en sujetos instruidos en las técnicas de cepillado. En estudios con sujetos no instruidos, hubo mejorías mínimas, a menudo no significativas; en otros estudios, con instrucción y períodos de cepillado supervisado, se produjo una mejoría en la eliminación de la placa. Generalmente se acepta que, aunque la instrucción en el cepillado mejora la eliminación de la placa, en pacientes colaboradores, se requiere una higiene oral profesional, para controlar la placa en pacientes poco proclives a cooperar. ${ }^{(13)}$

El Consejo de la Asociación Dental Americana, en Terapéutica Dental, ha recomendado un tiempo de cepillado de 3 minutos. Sin embargo, la mayoría de los individuos se cepillan sólo 60 segundos. Para evaluar los cepillos dentales, la mayoría de los investigadores emplean períodos de cepillado de 1 minuto, cuando el cepillado es supervisado, y periodos de cepillado de 2 minutos, cuando no es supervisado y se realiza en el domicilio. Está bien documentado que la eliminación de la placa aumenta con el tiempo de cepillado. ${ }^{(13)}$

Existen algunas situaciones en las que algunas personas se ven impedidas de realizar su aseo bucal, cuando se encuentran fuera de sus hogares, ya que no llevan consigo los instrumentos para realizarla, viéndose obligados a utilizar recursos emergentes no adecuados para sus dientes, como son los palillos u otros objetos. ${ }^{(3)}$
Considerando los aspectos mencionados, se decidió diseñar y evaluar un cepillo dental desechable y biodegradable, que pretende ser de fácil manejo y bajo costo, para ser utilizado fuera del hogar, con el fin de disminuir la placa bacteriana y además reforzar el hábito de higiene oral con los instrumentos adecuados, en cualquier lugar y momento.

\section{MATERIALES Y MÉTODOS}

\section{Diseño}

Para su elaboración y diseño se tuvieron en cuenta parámetros formales, funcionales, de uso, ergonómicos, de producción y legales.

Diseño formal: Como base se tomaron los diferentes aspectos formales que presentan los cepillos actuales: ubicación de las cerdas, tamaño del mango y tamaño de las cerdas.

Diseño funcional: El objetivo funcional del cepillo de dientes es ser un instrumento para la higiene oral; para ello se tuvieron en cuenta la flexibilidad de las cerdas, su resistencia y modo de asir el cepillo.

Diseño ergonómico: El producto se diseñó pensando en la mano del colombiano. Para ello se tuvieron en cuenta el tamaño, disposición de la mano al asir el cepillo, biomecánica del cepillo y factores usuario-producto, en cuanto a acomodo y ubicación del cepillo.

Sistema de producción: El producto se produce en un $100 \%$ de manera artesanal, desde la recolección de los sobrantes de madera, la selección de la misma, el rayado, el pulido, la perforación del sitio de las cerdas y la semilla, el acabado de la superficie con cera de abejas, el corte del hilo de fique, el agregado de la cera de abejas en el extremo de cada cerda, la instalación de las cerdas en el sitio anteriormente perforado y la impregnación de las cerdas con pasta dental.

Sistema de uso: Su utilización comienza en el momento de abrir el empaque. Se retirará junto con el manual de uso, leerán las instrucciones, se humedecerá y se usará para realizar la higiene oral. Al terminar, se lavará, se secará y se desechará o se guardará hasta encontrar un sitio adecuado para plantarlo. De esta manera se cumple el ciclo de vida y se preserva el material de que está elaborado (Figuras 1 y 2). 
Figura 1. Anverso del Manual de instrucciones para el uso del cepillo dental
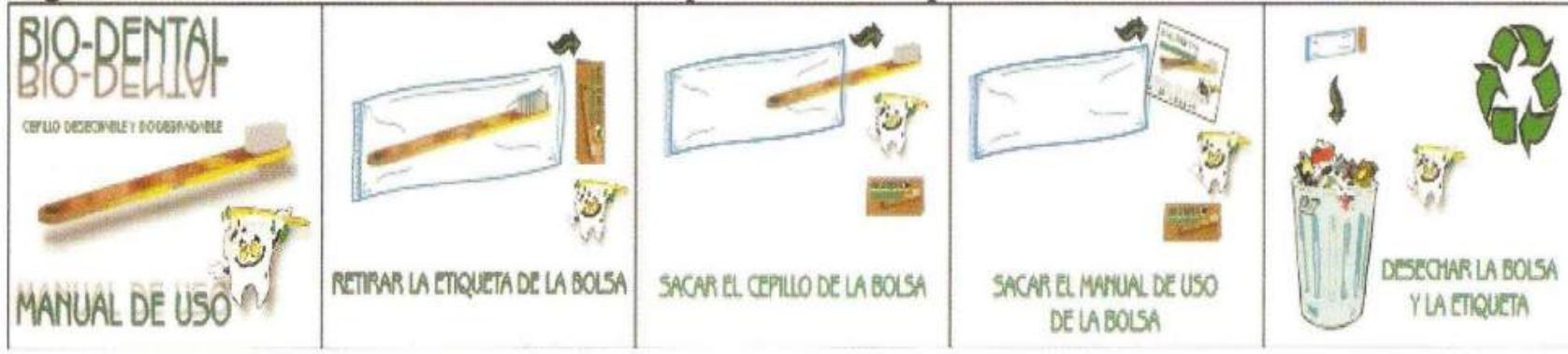

Figura 2. Reverso del Manual de instrucciones para el uso del cepillo dental

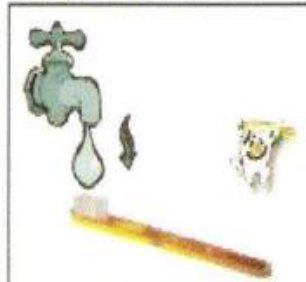

HUMEDECER EL CEPLO BOO-DETRL.

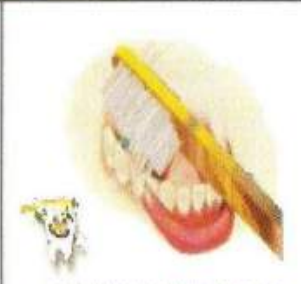

REALAR LA HIOETE ORAL

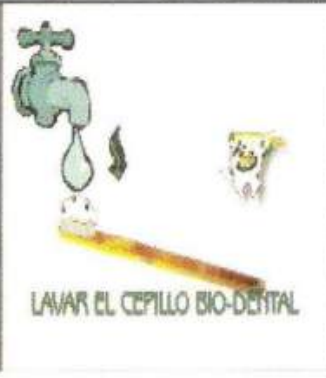

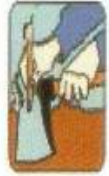

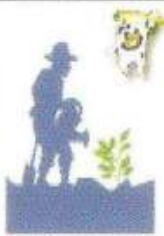

OPCOOH FHAL 2:

SEMBAREL CPILLO GIODETTAL PARA TERARE COCLO DE VDA

\section{Diseño e implementación de su desarrollo}

El cepillo lo conforman elementos totalmente biodegradables; su fabricación es netamente artesanal. El mango está hecho de madera. Tiene dos características específicas: en el primer tercio lleva un bajo relieve que se utiliza como lugar de apoyo del dedo pulgar en el momento de la higiene oral; en el extremo posterior se encuentra ubicado un receptáculo, que tiene en su interior una semilla de árbol maderable; como acabados lleva una película de cera de abejas sobre su superficie, con el fin de ocultar cualquier imperfección posible y así darle mayor suavidad en el momento del contacto. Las cerdas son de fibra natural, en este caso se utilizó hilo de fique; ya ensambladas las cerdas en el mango, se impregnan con crema dental (Figura 3).
El sistema de unión entre el mango y las cerdas está conformado por una estructura con perforaciones en las que se ubican las respectivas cerdas; para darle una fijación mayor, cada cerda en su extremo de contacto lleva cera de abejas. El empaque esta conformado por una bolsa de celofán, una pestaña en cartón y, como unión, hilo de fique.

Evaluación preliminar y exploración del mercado potencial: La investigación de mercados, para explorar el grado de aceptabilidad del nuevo producto, se llevó a cabo en la ciudad de Bucaramanga, tomando como mues-

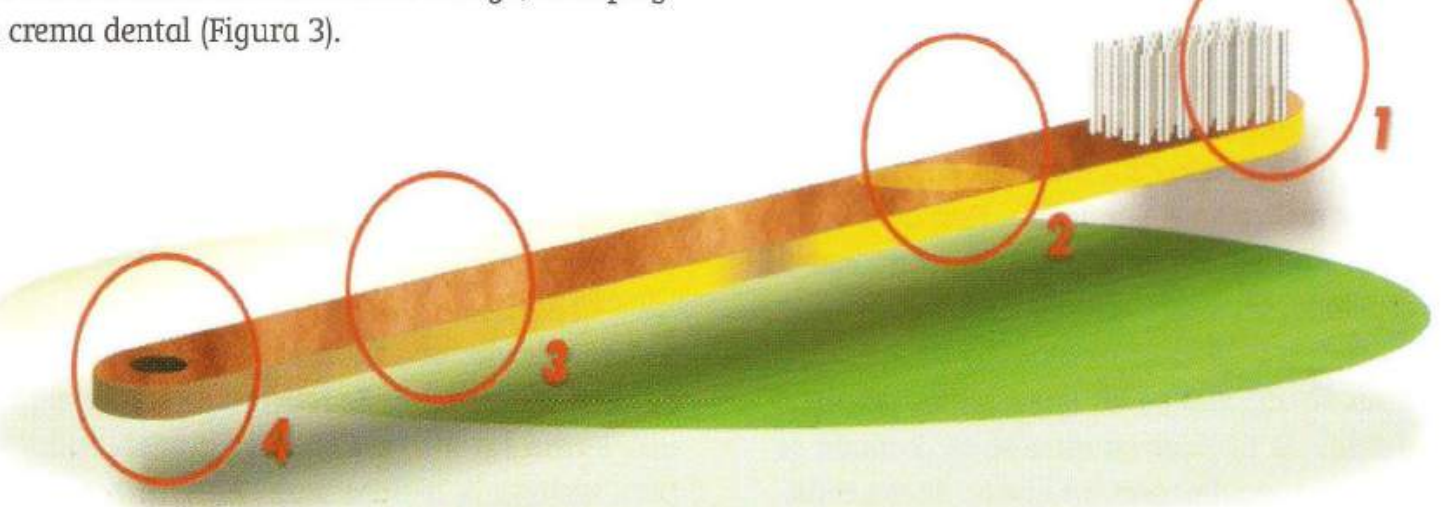

Figura 3. Características del diseño del cepillo dental

1. Cerdas en fique.

2. Lugar de ubicación del dedo pulgar.

3. Cuerpo en madera.

4. Receptáculo para alojar la semilla. 
tra treinta personas, entre los 20 y 27 años; de éstas, diez eran estudiantes de último semestre de odontología y veinte eran personas con diferentes ocupaciones. Para la muestra se elaboraron treinta cepillos.

Para realizar la investigación se tomaron dos días hábiles, en los que cada persona experimentaba el nuevo cepillo y diligenciaba la encuesta. Al terminar la higiene oral, cada encuestado depositó el cepillo en una bolsa, para dar curso a su aplicación ecológica, es decir, enterrarlo en tierra húmeda y fértil, donde la semilla cumplirá el proceso de germinación y desarrollo.

\section{RESULTADOS}

Al finalizar su utilización, los cepillos tenían un aspecto normal y sus cerdas conservaban su posición. En la Tabla 1 se destacan los resultados sobre la presentación y la

Tabla 1. Resultados en cuanto a presentación y utilidad del producto

\begin{tabular}{l|c|c|c}
\hline \multicolumn{1}{c|}{ Variable } & Calificación & Número & Porcentaje \\
\hline Presentación general & Regular & 7 & 23.3 \\
\hline & Buena & 17 & 56.7 \\
\hline Materiales & Excelente & 6 & 20 \\
\hline & Regular & 4 & 13.3 \\
\hline Cumplió sus expectativas en cuanto a higiene oral & Buena & 11 & 36.7 \\
\hline Los materiales proporcionan higiene y seguridad & Excelente & 15 & 50 \\
\hline Crema suficiente para limpieza & $\mathrm{Si}$ & 27 & 90 \\
\hline Aceptación general como producto ecológico & $\mathrm{Si}$ & 27 & 90 \\
\hline Tratamiento final & Excelente & 30 & 100 \\
\hline Lo conservaría para siembra & & & 23.3 \\
\hline Lo depositaría en la basura & $\mathrm{Si}$ & 27 & 90 \\
\hline Lo guardaría como souvenir & $\mathrm{Si}$ & 2 & 6.7 \\
\hline
\end{tabular}

Tabla 2. Precio y puntos de distribución

\begin{tabular}{c|c|c}
\hline Variable & Número & Porcentaje \\
\hline Precio justo considerando materiales y servicio prestado $(\$ 500.00)$ & 30 & 100 \\
\hline Estaría dispuesto a pagar \$ 500.00 & 30 & 100 \\
\hline Punto de distribución óptimo: & & \\
\hline Restaurantes & 29 & 96.7 \\
\hline Tiendas & 30 & 100 \\
\hline Supermercados & 27 & 90 \\
\hline Otros sitios de distribución: $\quad$ & \\
\hline Hoteles & 30 & 100 \\
\hline Tabernas & 28 & 93.3 \\
\hline Sitios de comidas rápidas & 30 & 100 \\
\hline Cines & 27 & 90 \\
\hline Teatros & 27 & 90 \\
\hline Establecimientos de recreación & 30 & 100 \\
\hline Sitio de trabajo & 30 & 100 \\
\hline Baños públicos & 30 & 100 \\
\hline Establecimientos educativos & 30 & 100 \\
\hline
\end{tabular}


Tabla 3. Recomendaciones y sugerencias.

\begin{tabular}{|c|c|c|}
\hline Recomendación & Número & Porcentaje \\
\hline \multicolumn{3}{|l|}{ Para su uso: } \\
\hline Demostraciones & 12 & 40 \\
\hline Ver manual de indicaciones & 16 & 60 \\
\hline \multicolumn{3}{|l|}{ Para comprarlo: } \\
\hline Campañas impulsadoras & 25 & 83.3 \\
\hline Conocer el producto & 5 & 16.7 \\
\hline \multicolumn{3}{|l|}{ Para promoverlo: } \\
\hline Hacer volantes & 10 & 33.3 \\
\hline Realizar charlas & 13 & 43.3 \\
\hline Comerciales en televisión & 3 & 10 \\
\hline Comerciales en radio & 4 & 13.3 \\
\hline \multicolumn{3}{|l|}{ Cambios que le haría al cepillo: } \\
\hline Ninguno & 22 & 73.3 \\
\hline Mejorar las cerdas & 5 & 16.6 \\
\hline Mejorar el mango & 3 & 10 \\
\hline
\end{tabular}

utilidad del producto. En la tabla 2 se presentan los resultados del producto en cuanto a precio y lugares de distribución; se aprecia, en general, una buena aceptación y disposición para su compra.

Finalmente la Tabla 3 describe las recomendaciones y sugerencias de los usuarios del producto. Se percibe un buen nivel de aceptación. En cuanto a la actitud del potencial consumidor del producto: 11 (36.6\%) lo comprarían, ya que lo consideran muy práctico; 14 (46.\%) lo consideran muy útil, y 5 (16.6\%) económico.

Cabe destacar, en general, la buena aceptación del cepillo dental biodegradable y la aceptación por el usuario como elemento de higiene oral y como estrategia ecológica para promover la reforestación según las cifras presentadas.

\section{DISCUSIÓN}

Alcance: El diseño y la evaluación del cepillo dental desechable y biodegradable sugieren que el nuevo producto podría convertirse en un instrumento útil, económico y de fácil aplicación para facilitar la higiene oral, fuera del hogar.

La adición de crema dental en las cerdas supera la dificultad de llevar aparte el tubo de pasta dentrifica; en un sólo producto el usuario encontrará los elementos necesarios para realizar su higiene oral.
El aporte del proyecto en los tiempos modernos enfatiza la cultura de la ecología y la calidad ambiental, aprovecha los materiales residuales de las empresas transformadoras de madera, junto con la utilización de materiales naturales como el fique. Esto mejora la vida útil de los materiales, considerando su tiempo de descomposición y retorno al ciclo biológico.

Limitaciones: Desde el punto de vista del diseño, en el momento de conformación y desarrollo del cepillo dental desechable y biodegradable, no se percibieron limitaciones, debido a que los materiales utilizados son flexibles y se dejan trabajar.

Los evaluadores no percibieron limitaciones aparentes, ya que conserva las características formales y funcionales, similares a las de los cepillos normales.

Sugerencias: Un pequeño grupo de evaluadores sugiere analizar el mango del cepillo en cuanto a longitud y analizar las cerdas en cuanto a su altura.

Al ampliar el estudio sobre el mercado del cepillo dental desechable y biodegradable se debe procurar la confección de diversos cepillos desechables, específicos para cada tipo de maloclusiones (clase I, clase II, clase III), y arcadas dentarias (ovaladas, en V, cuadradas); de esta manera se satisfacen las necesidades de los diferentes usuarios. 
El producto, por ser un elemento biodegradable y desechable, se constituye en una nueva fuente de vida colaborando con el ecosistema. Al ser sembrado, cumple dos funciones: convertirse en desecho orgánico y abono y proteger la semilla, mientras germina.

Desde el punto de vista técnico y considerando las recomendaciones de Heasman y col. (6), la evaluación de este nuevo prototipo, debe realizarse en forma estructurada; inicialmente con modelos in vitro para optimizar el diseño y posteriormente in vivo, con voluntarios a fin de evaluar su capacidad para remover la placa dental y su efecto abrasivo al utilizarlo a largo plazo.

\section{BIBLIOGRAFÍA}

1. Herazo B. Higiene bucodental y cepillos dentales. Editorial ECO. Bogotá. 1990

2. Fedi PF. The periodontic syllabus. 2nd ed Philadelphia, 1975.

3. Revista Federación Odontológica Colombiana No. 144. Aparatos Profilácticos Dentales de la comisión de productos dentales de la Federación Dental Internacional: 62 - 63 .

4. Dimosthenis M, Joss A, Christensen MM, Meng HX, Suvan JE, Lang NP. Comparison of the clinical effects and gingival abrasion aspects of manual and electric toothbrushes. J Clin Periodontol 2001; 28: 65 - 72.

5. Claydon N, Leach K, Newcombe RG, Ley F, Scratcher C, Addy M. The use of professional brushing to compare three toothbrushes for plaque removal from individuals with gingival recession. J Clin Periodontol 2000; 27: 749 - 752.

6. Heasman PA, McCracken GI. Powered toothbrushes: A review of clinical trials. J Clin Periodontol 1999; 26: 407 - 420.

7. Claydon N, Leach K, Addy M, Newcombe RG, Ley F, Scratcher C. Comparison of a double-textured prototype manual toothbrush with three branded products. A professional brushing study. J Clin Periodontol 2000; 27: 744 - 748 .

8. Renton-Harper P, Addy M, Newcombe RG. Plaque removal with the uninstructed use of electric toothbrushes: comparison with a manual brush and toothpaste slurry. J Clin Periodontol 2001; 28: 325 - 330.

9. Dyer D, Addy M, Newcombe RG. Studies in vitro of abrasion by different manual toothbrush heads and a standard toothpaste. J Clin Periodontol 2000; 27: 99 - 103.

10. Khocht A, Simon G, Person P, Denepitiya JL. Gingival recession in relation to history of hard toothbrush use. J Periodontol 1993; 64: 900 - 905 (abstract).
11. Checchi L, Minguzzi S, Franchi M, Fosteleoni G. Toothbrush filaments end-rouding: stereomicroscope analysis. J Clin Periodontol 2001; 28: $360-364$

12. Saxer UP, Yankell SL. Impacto de los cepillos dentales perfeccionados sobre las enfermedades dentales (I) Quintessence 1998; 11: $485-497$.

13. Soxer UP, Yankell SL. Impacto de los cepillos dentales perfeccionados sobre las enfermedades dentales (II) Quintessence 1998; 12: $505-510$. 